\title{
Bleisaum in Indien
}

Ein 18-Jähriger suchte in Neu Delhi in Indien wegen seit einer Woche bestehender kolikartiger Bauchschmerzen die Nothilfe auf. Stuhlgewohnheiten und Blasenfunktion waren nicht verändert, er war Nichtraucher. Im vorhergehenden Jahr hatte der Mann in einer Firma gearbeitet, in der Batterien wiederverwertet wurden.

$B_{d}^{\text {eider }}$ i der Untersuchung zeigte sich ein diffuser Druckschmerz im Bereich des Abdomens und eine blauschwarze Verfärbung am Zahnfleisch. Die Routinelaborparameter und die Oberbauchsonografie ergaben keinen pathologischen Befund. Allerdings war die Bleikonzentration im Blut mit $81 \mathrm{ng} / \mathrm{dl}$ (Normalbereich $<10$ ng/dl) mehr als achtfach erhöht. Der Patient wurde stationär aufgenommen und einer Chelattherapie mit Penicillamin unterzogen. Bei der Entlassung zwei Wochen später waren die Bauch- schmerzen und die Bleikonzentration im Blut zurückgegangen. Auch der Bleisaum am Zahnfleisch war verschwunden. Man beriet den Mann bezüglich einer möglichen Intoxikation an seiner Arbeitsstelle. Welche Folgen das für den Arbeitgeber hatte, wird nicht mitgeteilt.

Kommentar: Bleivergiftungen sind in Entwicklungsländern nicht selten und kommen meistens bei den Beschäftigten von Firmen vor, die Batterien und Plastikschrott verarbeiten oder Kontakt zu

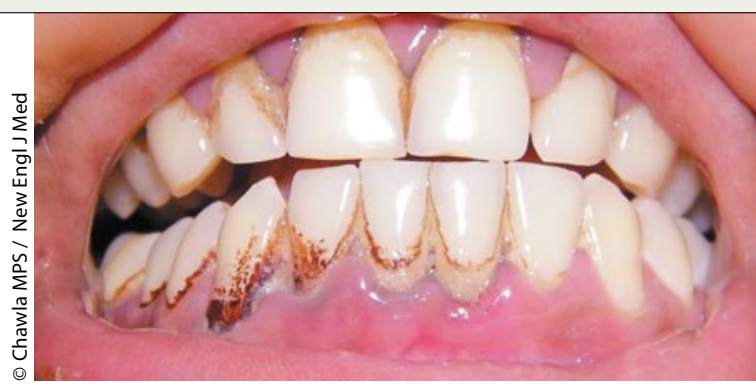

Burton's line (Bleisaum) bei einem 18-jährigen indischen Patienten.

Druckerschwärze, bleihaltigen Farben oder Keramikprodukten haben. Die giftigen Abfälle der Industriestaaten werden oft auf Kosten der Gesundheit der Bevölkerung in den Entwicklungsländern entsorgt. Henry Burton konnte seine Beobachtung des Bleisaums (Burton's line) Anfang des 19. Jahrhunderts noch in England machen. Prof.Dr. Hermann FüeßI

Chawla MPS, Sdriyal D. Images in clinical medicine. Burton's line. New Engl J Med 2012; 367: 937

\section{Europäische Leitlinien zur Vitiligo-Therapie}

\section{Leitlinien für die Behandlung einer Vitiligo existierten bislang nur auf nationaler Ebene in Großbritannien. Ein internationales Team hat nun europäische S1-Empfehlungen publiziert.}

n der Leitlinie konnten Experten aus zahlreichen Ländern, darunter auch Markus Böhm aus Münster, den aktuellen Stand der Evidenz zusammentragen.

Bei gesicherter Diagnose wird die Kontrolle von Schilddrüsenparametern empfohlen. Liegen Hinweise auf weitere Autoimmunerkrankungen vor, sollten zusätzliche Tests zur Abklärung erfolgen.

Besteht keine diagnostische Sicherheit, eignen sich Stanzbiopsien sowie beispielsweise mykologische oder molekularbiologische Verfahren zur Abklärung.

Segmentale und begrenzte nicht segmentale Vitiligo: An erster Stelle steht das Vermeiden von Triggerfaktoren. Zur lokalen Therapie werden Kortikosteroide oder (mit weniger Nebenwirkungen) Calcineurin-Inhibitoren empfohlen. Aufgrund der noch dünnen Datenlage sollte die Behandlung mit Calcineurininhibitoren auf die Kopf- und Halsregion beschränkt bleiben. Die Präparate werden zweimal am Tag, möglichst unter täglicher moderater Sonnenexposition, aufgetragen. Bei erfolgrei- cher Behandlung wird die Therapie über zwölf Monate forgeführt. Müssen auf große Hautareale über längere Zeit Kortikosteroide appliziert werden, sollten Substanzen wie Mometasonfuroat oder Methylprednisolonaceponat zur Anwendung kommen. Als optimal wird ein diskontinuierliches Behandlungsschema mit Anwendung an 15 Tagen pro Monat über sechs Monate empfohlen. Ist eine kontinuierliche Behandlung nötig, raten die Autoren zur Applikation einmal täglich über maximal drei Monate.

Als Second-Line-Therapie kommt eine Schmalband-UVB-Therapie, insbesondere mit dem Excimer-Laser, infrage. Als letzte Möglichkeit gelten chirurgische Eingriffe, etwa eine Eigenhaut- oder Melanozyten-Transplantation.

Nicht segmentale Vitiligo: Auch hier sind zunächst Triggerfaktoren auszuschalten. Mit einer Schmalband-UVBTherapie über mindestens drei Monate ist möglicherweise eine Stabilisierung zu erreichen. Sind mehr als 15-20\% des Körpers betroffen und breitet sich die Pig- mentstörung aktiv aus, empfehlen die Autoren eine Ganzkörperbestrahlung. Ist die Behandlung wirksam, sollte sie über mindestens neun Monate und maximal zwei Jahre fortgesetzt werden. Eine Kombination der Bestrahlung mit systemischen und/oder topischen Therapien ist möglich.

Als Zweitlinien-Therapie kommen systemische Steroide oder bei schnellem Fortschreiten bzw. erfolgloser Stabilisierung unter Schmalband-UVB ggf. auch Immunsuppressiva zum Einsatz. Tritt keine Besserung ein, sind an geeigneten Körperstellen bedingt auch Transplantationen möglich.

Bleibt der Behandlungserfolg aus und erstrecken sich die Pigmentstörungen zu $50 \%$ über gut sichtbare Bereiche, ist zuletzt eine Depigmentation der restlichen dunkleren Haut zu diskutieren.

Fazit: Zum ersten Mal ist eine VitiligoLeitlinie auf europäischer Ebene verfügbar. Neben den genannten Empfehlungen legen die Autoren Wert auf eine ausführliche Planung der Therapie zusammen mit dem Patienten. Auch eine psychologische Unterstützung betroffener Patienten ist essenziell. Dr. ChristineStarostzik

Taieb A et al. Guidelines for the management of vitiligo: the European Dermatology Forum consensus. Br J Dermatol 2013; 168: 5-19 